\title{
High Speed On-Chip Signal Generation for Debug and Diagnosis
}

\author{
Tsung-Yen Tsai • Sadok Aouini • \\ Gordon Walter Roberts
}

Received: 14 October 2011 / Accepted: 1 March 2012 / Published online: 1 April 2012

(C) The Author(s) 2012. This article is published with open access at Springerlink.com

\begin{abstract}
This article presents methods and circuits for synthesizing test signals in the time/frequency domain. An arbitrary signal is first encoded using sigma-delta modulation in the digital amplitude-domain and converted to the time or frequency domain through a digital-to-time converter (DTC) or digital-to-frequency converter (DFC) operation realized in software. In hardware, the resulting bit-stream is inputted cyclically to a high-order phase-locked loop (PLL) behaving as a time-mode reconstruction filter in the appropriate domain (time or frequency). A high-speed prototype implementation consisting of a 4 th order PLL built in $0.13 \mu \mathrm{m}$ complementary metal oxide semiconductor (CMOS) process with an off-chip loop filter has been fabricated and used to generate signals at $4 \mathrm{GHz}$. The digital nature and portability of the phase/ frequency test signal generation process makes the proposed scheme compatible with the IEEE 1149.1 test bus standard and easily amenable to any testing environment: production, characterization, design-fortest (DFT), or built-in self-test (BIST).
\end{abstract}

Keywords Analog test - Mixed-signal test • Design-for-test - Built-in self-test - Phase generation • Frequency synthesis - Sigma-data encoding • Integrated circuit $\cdot$ Phase-locked loop

Responsible Editor: D. Keezer

T.-Y. Tsai · S. Aouini · G. W. Roberts $(\bowtie)$

Integrated Microsystems Laboratory,

Department of Electical \& Computer Engineering,

McGill University, 3480 University Street,

Montreal, QC, Canada H3A 2A7

e-mail: gordon.roberts@mcgill.ca

\section{Introduction}

The ability to generate high-frequency test signals onchip that can be made to vary over frequency and phase under external program control provides a useful debug and diagnosis tool (Fig. 1). As operating frequencies and pin counts rise, it is increasingly difficult and costly to route high-speed signals on and off-chip. Many factors need to be considered, such as impedance matching, mismatch uncertainty, crosstalk, and clock skew among others, requiring extensive analysis. Also, adding pins in an already crowded package exclusively for testing may not be acceptable in all situations. As such, design-for-test (DFT) techniques that use circuitry already available on-chip is preferable.

Traditionally, information has been processed and encoded in the voltage/amplitude domain; however, more recently the encoding of information in time has gained considerable popularity [23] and [6]. Time mode signal processing involves encoding the information in the form of time difference variables using phase modulation. A digital-to-time converter (DTC) can be seen as any device used to map a digital value to a timebased signal, similar to a digital-to-analog converter (DAC) in the voltage/amplitude domain. Likewise a digital-to-frequency converter (DFC) converts a digital code to a corresponding instantaneous frequency. From a system perspective, such a conversion process should always be combined with a reconstruction filter to eliminate the images in the output spectrum. In [2], it has been shown that a phase-locked loop can be used as a reconstruction filter for DTCs.

In this work we limit our discussion to the generation of high-frequency signals on-chip under external program control. As depicted in Fig. 2, the phase/frequency 
Fig. 1 System

implementation of on-chip high-frequency generator utilizing the 1149.1 test bus standard

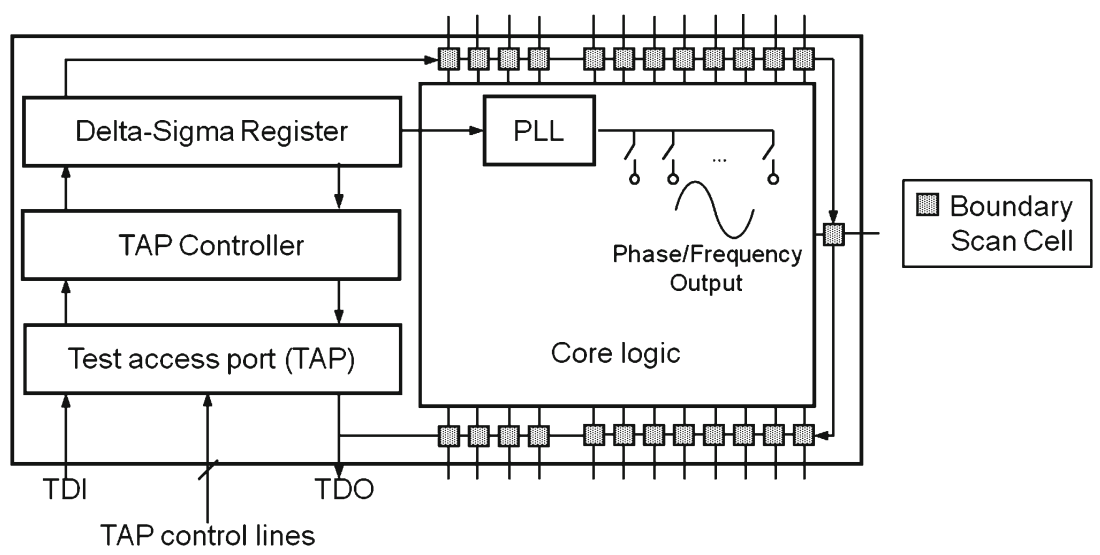

signal generation process consists of first encoding a DC signal using sigma-delta modulation. Sigma-delta modulators are basically oversampling analog-todigital converters, meaning that the sampling rate of the signal is increased to the point where a low-resolution quantizer is enough for accurate digitization. Then, the sigma-delta encoded digital signal is converted to the time or frequency domain through a DTC or DFC process. Finally, a PLL is used as a time/frequency domain analog reconstruction filter. The hardware implementation consists of only a periodic bit-stream containing the sigma-delta encoded phase or frequency signal and a PLL. In fact, it can easily be incorporated within a DFT or BIST framework, such as that described in [18], or in a boundary-scan compliant scheme [8], as depicted in Fig. 1. Using an existing BIST framework allows costs to be lowered, as the IEEE 1149.1 test bus allows the sigma-delta bitstream to be routed to an already existing PLL, then the output can be routed to a device under test. This lowers both the cost and difficulty of implementation.

The test implementation as presented is appropriate for testing analog circuitry. For testing of digital circuits, proper buffering and/or level shifting may be required. As the PLL in this implementation is used as a time domain filter, any PLL with the appropriate bandwidth may be used. PLLs have been demonstrated

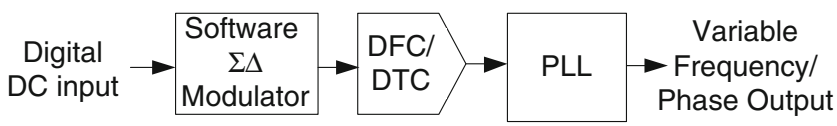

Fig. 2 Phase/frequency test signal generation process where a DC signal is encoded using sigma-delta modulation, converted to phase or frequency through a DTC or DFC, and reconstructed using a PLL serving as time/frequency domain filter to be built in $90 \mathrm{~nm}$ and $45 \mathrm{~nm}$ technologies; as such, the technique should be fully compatible with them. As this technique uses a bitstream and a PLL, it is scalable with technology and a higher speed implementation would be realizable.

This paper is divided as follows: first, the phase and frequency encoding process using sigma-delta modulation is described in Section 2. In Section 3, MATLAB/ Simulink simulations are used to validate the technique. In Section 4, the design of a prototype phase/frequency test signal generator incorporating a custom high-speed PLL built in CMOS $0.13 \mu \mathrm{m}$ and running at $4 \mathrm{GHz}$, and custom PCB used to interface the PLL chip to the test equipment are described. The experimental results are then outlined in Section 5 and finally, conclusions are drawn and future potential avenues are discussed in Section 6.

\section{Phase and Frequency Encoding Using Sigma-Delta Modulation}

In this section, an overview of the phase encoding process using sigma-delta modulation and digital-totime conversion techniques is presented and then extended to frequency encoding using digital-tofrequency conversion.

\subsection{Phase Encoding}

A digital input can be converted to a phase modulated signal through a digital-to-time conversion process as described in [1]. Referring to Fig. 3, the process is as follows: a parallel multi-bit digital input is applied to the input of a DTC and a serial output with the corresponding time delay is generated. The mapping 


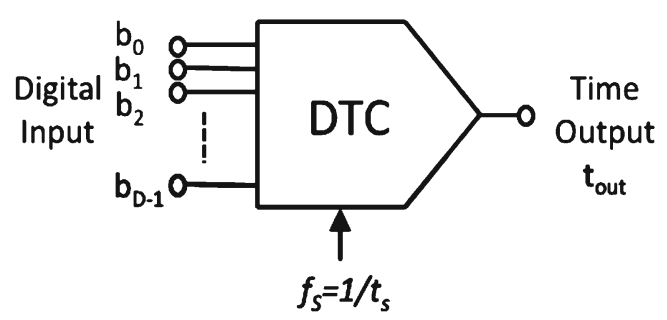

Fig. 3 A D-bit digital-to-time converter showing digital input gives a corresponding time output

process between the input bits and the output time signal is assumed to be represented by

$t_{\mathrm{out}}=t_{\mathrm{ref}}\left(b_{0}+b_{1} 2^{1}+b_{2} 2^{2}+\ldots+b_{N-1} 2^{D-1}\right)+t_{\mathrm{os}}$,

where $t_{\text {out }}$ is the time output, $t_{\text {ref }}$ the reference time, $t_{\mathrm{os}}$ a time offset and $b$ the digital input.

A way to understand the DTC mapping process is to look at the output sequence in terms of the input sequence, as illustrated in Fig. 4. In the case of a 1-bit DTC having a 90-degree phase encoding range, every ' 0 ' value in the digital domain is mapped to the bit sequence ' 1100 ' and every ' 1 ' is mapped to the sequence '0110'. Note here that the same concept can be extended to a multi-bit, different phase range and duty cycle encoding. In this case, to preserve the sampling frequency, the output phase code should be clocked four times faster than the input amplitude code. The PLL will also lock on to this sampling frequency, as it is the carrier frequency for this phase modulation scheme.

Digital-to-time conversion can be combined with sigma-delta modulation to digitally encode a phase signal, as depicted in Fig. 2. The amplitude domain

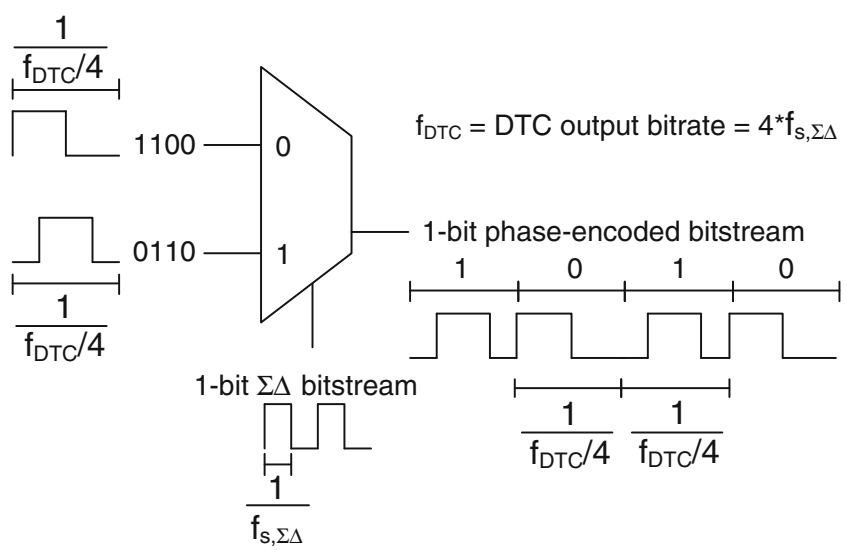

Fig. 4 Illustrating the phase encoding process for a 1-bit DTC having a 90 -degree phase encoding range sigma-delta modulator followed by the DTC is equivalent to a sigma-delta modulation process occurring in phase. Hence, all the parameters of the sigma-delta modulator are mapped in a one-to-one correspondence to the phase domain. The modulator order, bandwidth and SNR should be equivalent in both domains. Thus, the maximum value of the sigma-delta modulated signal in the amplitude domain, $\Sigma \Delta_{\mathrm{MAX}}$, is mapped to the maximum phase shift $\phi_{\mathrm{MAX}}$; likewise, the minimum value of the sigma-delta modulated signal in the amplitude domain, $\Sigma \Delta_{\mathrm{MIN}}$, is mapped to the minimum phase shift $\phi_{\text {MIN }}$, without loss of generality if it is encoded using a single or multi-bit conversion. The amplitude to phase mapping coefficient is defined as

$\alpha_{\phi}=\frac{\phi_{\mathrm{MAX}}-\phi_{\mathrm{MIN}}}{\Sigma \Delta_{\mathrm{MAX}}-\Sigma \Delta_{\mathrm{MIN}}}\left[\frac{\mathrm{rads}}{V}\right]$

This equation defining $\alpha_{\phi}$ can also be seen as taking the full-scale range of the DTC over the full-scale range of the sigma-delta converter. In addition, it must be noted that an offset term $\phi_{\text {os }}$ can also be present to link the output instantaneous phase $\phi_{\text {out }}$ and the DTC input, denoted as $D T C_{\text {in }}$, as given by

$\phi_{\text {out }}=\alpha_{\phi} D T C_{\text {in }}+\phi_{\text {os }}$

It can be noted here that Eqs. 1 and 3 are related. In fact, in Eq. 1 we can convert $t_{\text {ref }}$ to the phase domain by multiplying it by $\omega_{S}\left(\omega_{S}=2 \pi / t_{S}\right)$, where $t_{S}$ is sampling period of the DTC as shown on Fig. 1 to give $\alpha_{\phi}$. Likewise $t_{\mathrm{os}}$ of Eq. 1 can be converted to $t_{\mathrm{os}}$ by the same relationship.

Since the DTC relates an input amplitude to a corresponding output phase signal by multiplying it by $\alpha_{\phi}$, the spectrum of the DTC output signal can be written in terms of the sigma-delta PSD output as (Fig. 5)

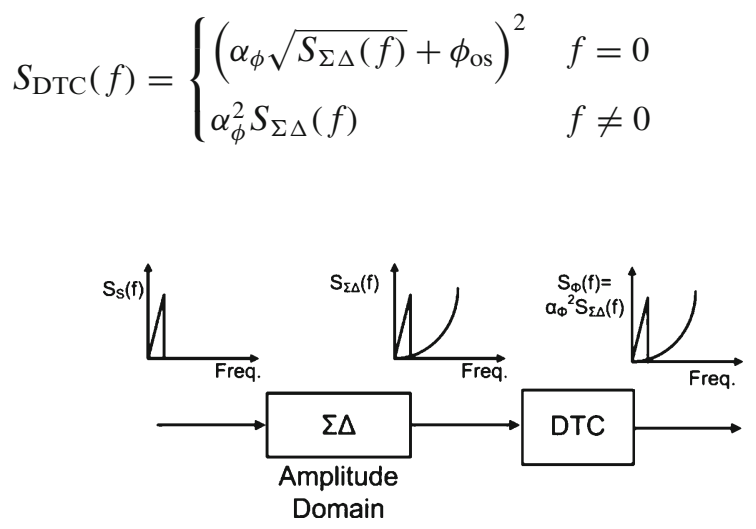

Fig. 5 PSD mapping from the amplitude domain through the DTC block to the phase domain 
Given that the PSD of the sigma-delta output can then be decomposed into a signal and noise component $S_{S, \Sigma \Delta}(f)$ and $S_{N, \Sigma \Delta}(f)$, respectively, this allows us to write the PSD of the DTC output as (for $f \neq 0$ )

$$
S_{\mathrm{DTC}}(f)=\alpha_{\phi}^{2} S_{S, \Sigma \Delta}(f)+\alpha_{\phi}^{2} S_{N, \Sigma \Delta}(f)
$$

It can be observed that a carefully designed phasefiltering function, implemented by the PLL, must be realized to properly filter the out-of-band quantization noise. To ensure that the in-band noise level is negligible, the phase-filtering function of the PLL must at least match the bandwidth and have a higher order than the sigma-delta modulator. Consequently, the PLL phase transfer function must be designed accordingly. However, just as for the amplitude domain, if the signal encoded using sigma-delta modulation has a smaller bandwidth than the modulator, the order of the filtering function of the PLL can be relaxed by also lowering its bandwidth. The SNR of the overall process can then be found using the terms above and leading to show that the SNR in the phase domain is indeed equal to the one in the amplitude domain, i.e.,

$S N R_{\phi}=\frac{P_{S, \phi}}{P N, \phi}=\frac{\alpha_{\phi}^{2} \int_{0}^{f_{B}} S_{S, \Sigma \Delta}(f) d f}{\alpha_{\phi}^{2} \int_{0}^{f_{B}} S_{N, \Sigma \Delta}(f) d f}=S N R_{\Sigma \Delta}$

\subsection{Frequency Encoding}

Just as for the DTC, the DFC is used to convert a digital input signal to a corresponding frequency. The general equation relating the input bits and instantaneous output frequency can be written as

$$
f_{\text {out }}=f_{\text {ref }}\left(b_{0}+b_{1} 2^{1}+b_{2} 2^{2}+\ldots+b_{N-1} 2^{D-1}\right)+f_{\text {os }}
$$

where $f_{\text {out }}$ is the time output, $f_{\text {ref }}$ the reference time, $f_{\text {os }}$ a time offset and $b$ the digital input.

Once again, recognizing that the operation of the DFC is to take as input a bit-stream consisting of D-bit words and create as output a 1-bit bit-stream whereby each input word is mapped to a corresponding sequence of bits representing a particular frequency component. For example, for a 1-bit DFC consisting of two frequencies, $f_{1}$ and $f_{2}$ where $f_{2}=2 * f_{1}$, the operation of the DFC is to convert a logical ' 0 ' input bit to a ' 1100 ' output sequence, and a logical ' 1 ' input bit to a '1010' output sequence, at a clock rate four times the original bit stream clock rate. The mapping algorithm is demonstrated in Fig. 6.

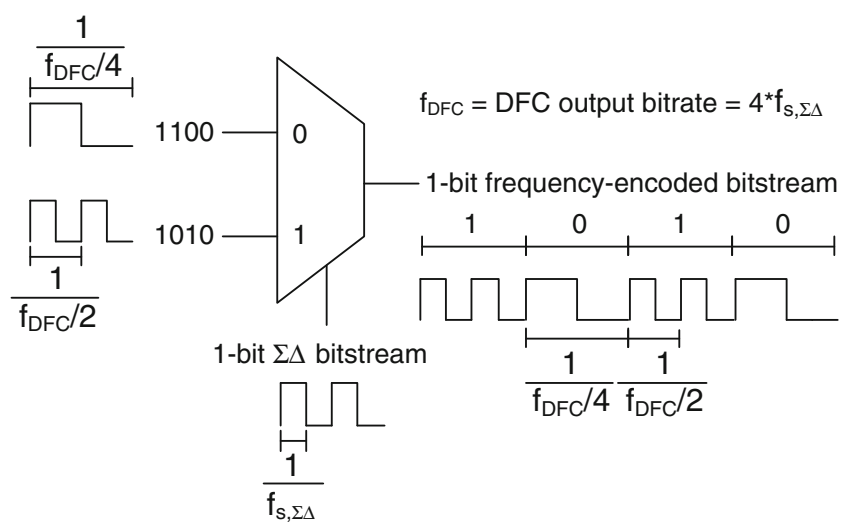

Fig. 6 Amplitude-to-frequency mapping where every ' 1 ' value is mapped to a frequency twice the rate of a ' 0 ' value

Just as in for phase synthesis, the same could be said for frequency synthesis. With regards to the DFC process, the sigma-delta bits are mapped to instantaneous frequencies. Here, the maximum value of the sigma-delta modulated signal in the amplitude domain, $\Sigma \Delta_{\mathrm{MAX}}$, is mapped to the maximum frequency $f_{\mathrm{MAX}}$; likewise, the minimum value of the sigma-delta modulated signal in the amplitude domain, $\Sigma \Delta_{\mathrm{MIN}}$, is mapped to the minimum frequency $f_{\mathrm{MIN}}$, without loss of generality if it is encoded using a single or multi-bit conversion. Here again, a mapping coefficient between the amplitude and frequency domain can be defined as

$\alpha_{f}=\frac{f_{\mathrm{MAX}}-f_{\mathrm{MIN}}}{\Sigma \Delta_{\mathrm{MAX}}-\Sigma \Delta_{\mathrm{MIN}}}\left[\frac{H z}{V}\right]$

Note that $\alpha_{f}$ can be seen as being the full-scale range of the DFC divided by the full-scale range of the sigmadelta modulator. In addition, it must be noted that an offset term $f_{o s}$ can also be present when linking the output frequency $f_{\text {out }}$ and the DFC input $D F C_{\text {in }}$ as given by

$f_{\text {out }}=\alpha_{f} D F C_{\text {in }}+f_{\text {os }}$

It can be noted here that Eqs. 7 and 9 are related. In fact, in Eq. 7, $f_{\text {ref }}$ can be expressed as $\alpha_{f}$. Likewise $f_{\text {os }}$ in both equations are actually the same.

The spectrum of the DFC output signal can then be written like before in terms of the sigma-delta PSD output as

$S_{\mathrm{DFC}}(f)= \begin{cases}\left(\alpha_{f} \sqrt{S_{\Sigma \Delta}(f)}+f_{\mathrm{os}}\right)^{2} & f=0 \\ \alpha_{f}^{2} S_{\Sigma \Delta}(f) & f \neq 0\end{cases}$ 


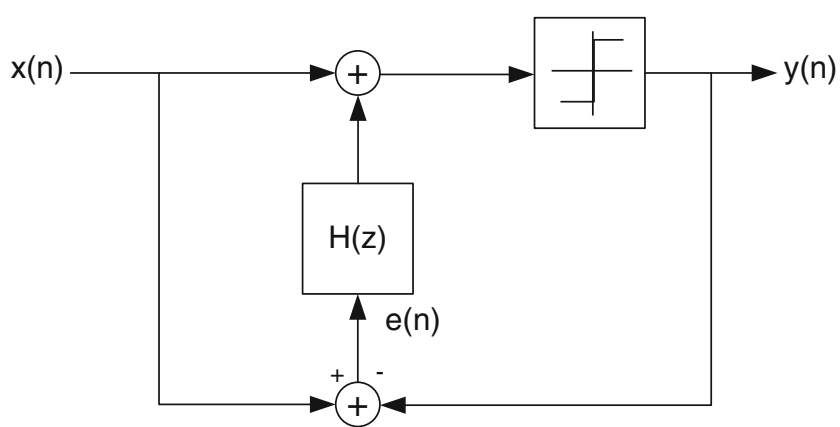

Fig. 7 General sigma-delta modulator structure

which we can then write in a more detailed form (for $f \neq 0)$ as

$$
S_{\mathrm{DFC}}(f)=\alpha_{f}^{2} S_{S, \Sigma \Delta}(f)+\alpha_{f}^{2} S_{N, \Sigma \Delta}(f)
$$

Assuming the quantization noise carried over from the sigma-delta encoding process is removed by a filtering function realized by the PLL, one can also show, following the previous subsection arguments, that the SNR of the DFC process has the same SNR as established by the sigma-delta encoding process.

\section{MATLAB Modelling and Simulation Results}

A third-order sigma-delta modulator was implemented in MATLAB. This allows for relatively quick simulation and verification of any changes made to the modulator, such as bandwidth or sampling frequency. The modulator was designed with a sampling frequency

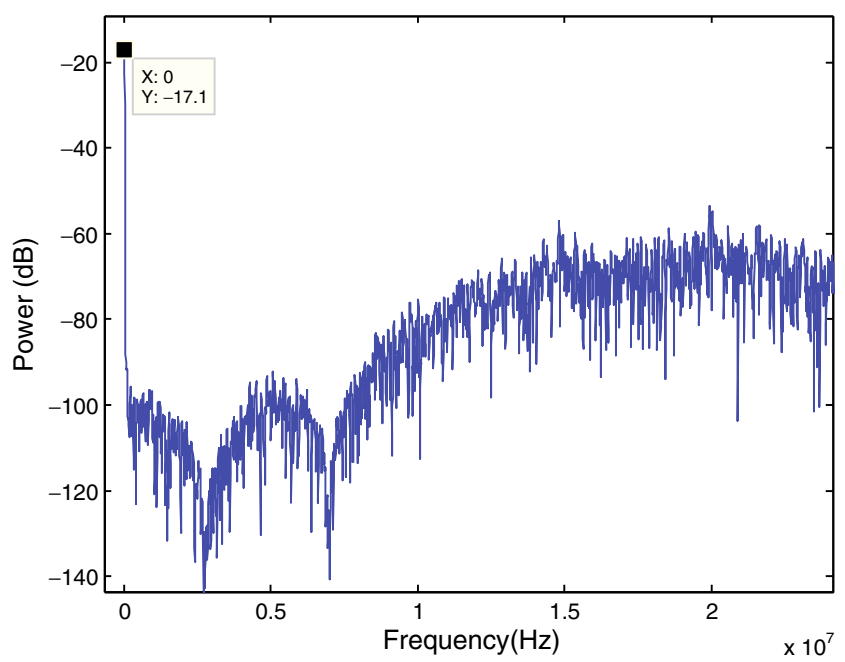

Fig. 8 Output PSD of sigma-delta modulator with 0.434 DC input

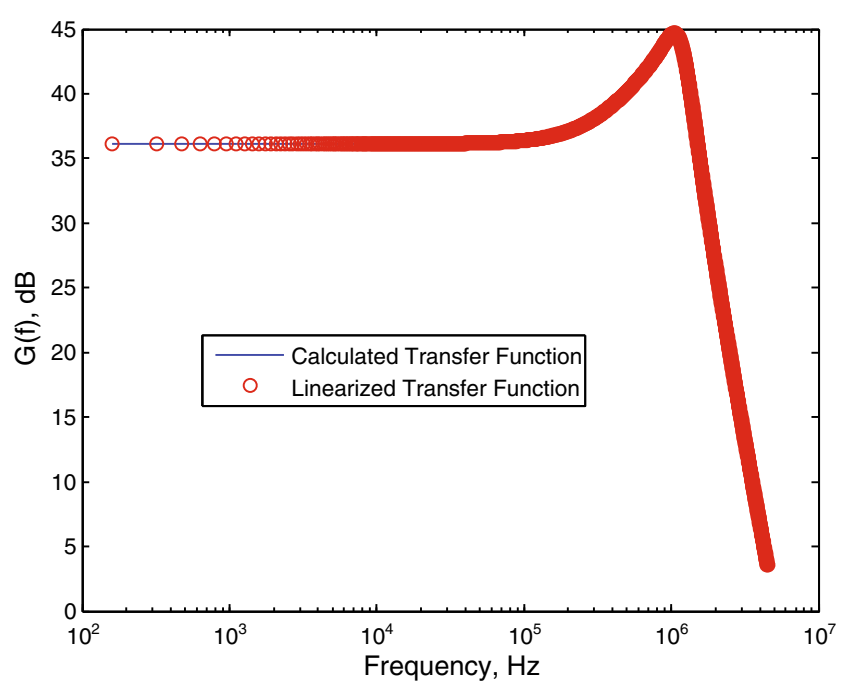

Fig. 9 PLL closed-loop transfer function

of $65 \mathrm{MHz}$, an OSR of 16, resulting in a bandwidth of approximately $2 \mathrm{MHz}$. It was designed using DSMOD [5] and simulated in Simulink using the general model shown in Fig. 7. The output PSD of the modulator is shown in Fig. 8. A Kaiser window with beta of 30 was used. The marker designates the input DC level (0.434).

The closed-loop response of the PLL can be seen in Fig. 9. The calculated transfer function (solid line) is compared with the transfer function of a linearized model (line with "O" markers) from Simulink. It can be seen that the two match exactly. The loop filter response is plotted in Fig. 10, with the calculated transfer function in solid and the linearized model with "O" markers. It can be observed that a pole at DC is present.

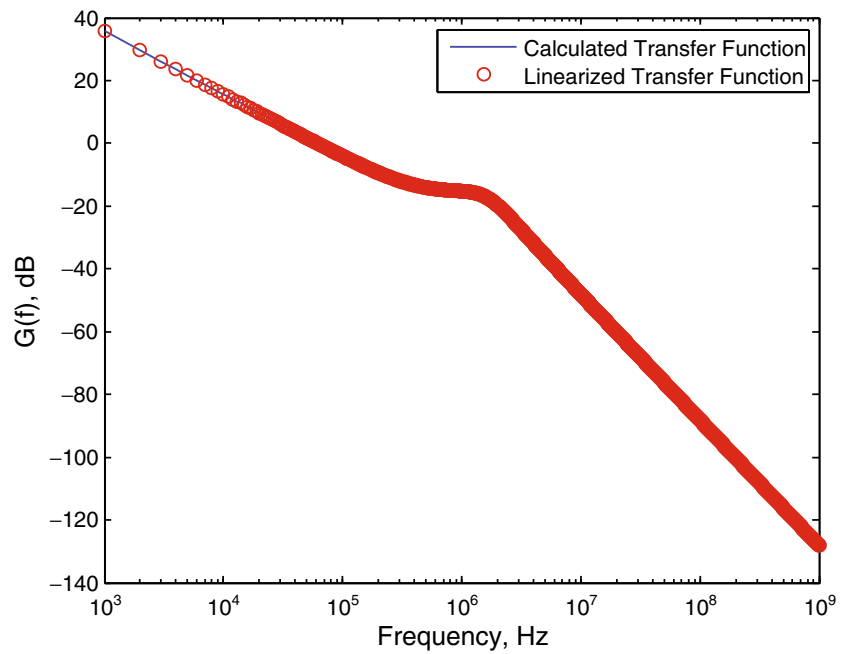

Fig. 10 PLL loop filter transfer function 


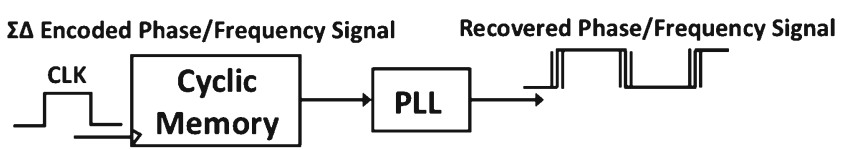

Fig. 11 Implementation of phase/frequency synthesis system

\subsection{System Simulation}

Figure 11 outlines the implementation of the phase/ frequency synthesis system. Going back to Fig. 2, the hardware implementation consists of a cyclic memory with the sigma-delta encoded phase signal applied to a high-order PLL behaving as a time-domain filter to eliminate the quantization noise. Thus, if the PLL bandwidth also matches that of the sigma-delta modulator, the encoded phase signal can accurately be recovered. The sampling frequency of the DFC is $180 \mathrm{MHz}$; correspondingly, the two encoded frequencies are 45 and $90 \mathrm{MHz}$, while the DTC is sampled at $260 \mathrm{MHz}$, which results in a carrier at $65 \mathrm{MHz}$. As shown in Fig. 11, the output of the DFC/DTC is stored in memory and repeated so that it appears as a constant, uninterrupted bitstream.

The frequency synthesis system was simulated with Simulink for three different DC input conditions; specifically, $0.434,0.452$, and 0.470 on a sigma-delta output scale of 0 to 1 . The corresponding output spectrums are superimposed on the plot shown in Fig. 12. As is evident, the PLL is locked at $4.13 \mathrm{GHz}, 4.18 \mathrm{GHz}$ and $4.23 \mathrm{GHz}$. These frequencies correspond exactly as that calculated by Eq. 9 when scaled by the PLL divider ratio of 64 . Likewise, the phase synthesis system

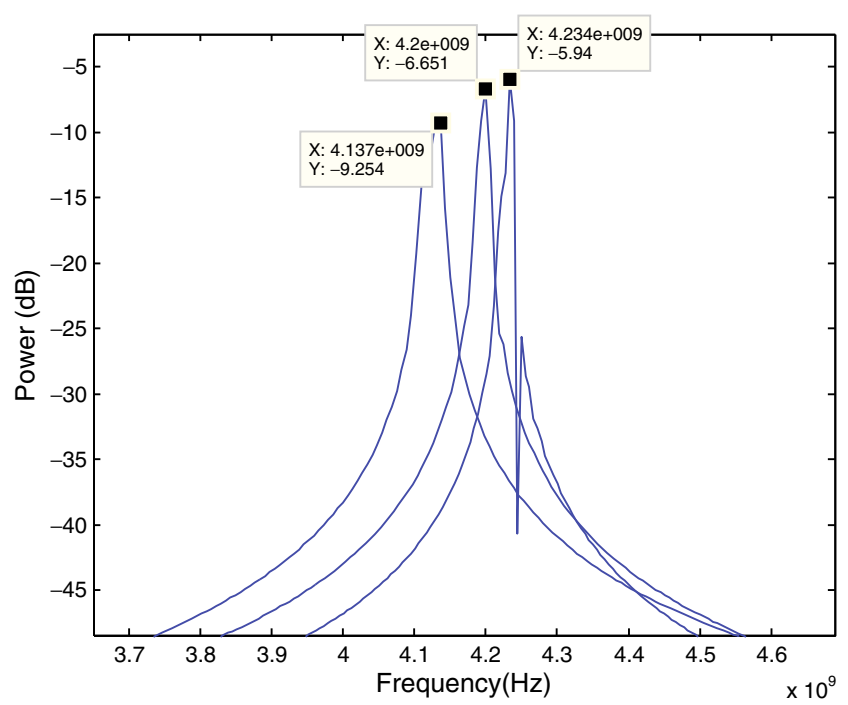

Fig. 12 Three tones produced by the frequency generator

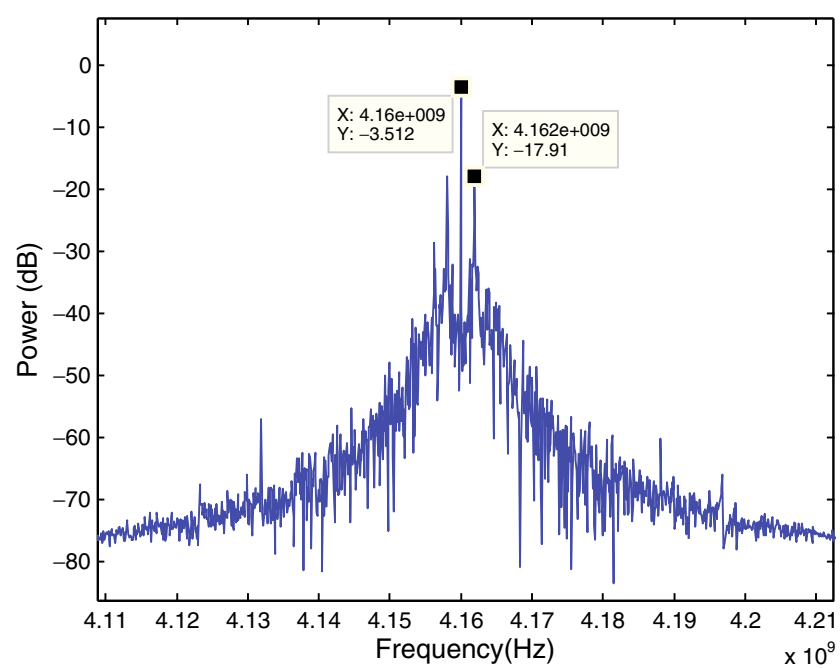

Fig. 13 Output spectrum of $0.01 \mathrm{amp}, 2 \mathrm{MHz}$ phase-encoded signal with $4.16 \mathrm{GHz}$ carrier

was simulated with a 0.01 amplitude, $2 \mathrm{MHz}$ sinusoidal input. The output spectrum of the PLL can be found in Fig. 13, where a $2 \mathrm{MHz}$ sideband is centred around a $4.16 \mathrm{GHz}$ carrier $(65 \mathrm{MHz} \times 64)$.

\section{Phase/Frequency Generator Implementation}

The phase/frequency generator consists of a cyclic memory element, containing a phase or frequency encoded sigma-delta bitstream, and a custom phaselocked loop whose bandwidth corresponds to that of the software-based sigma-delta modulator. This system is shown in Fig. 11. The details of both the cyclic memory and the design of the custom PLL are described, as well as the design of a PCB that allows the PLL to interface with test equipment.

\subsection{Cyclic Memory}

The phase/frequency generator requires a cyclic memory element in order to present an uninterrupted phase or frequency encoded bitstream to the PLL. This is accomplished by using an external pattern generator, further described in the Experimental Results section. Any programmable memory element can be used for this purpose (e.g., a FPGA).

\subsection{Custom PLL Design}

In order to test frequency and phase synthesis at high speeds, a custom PLL had to be designed and built. A top-down design methodology was employed to impose 


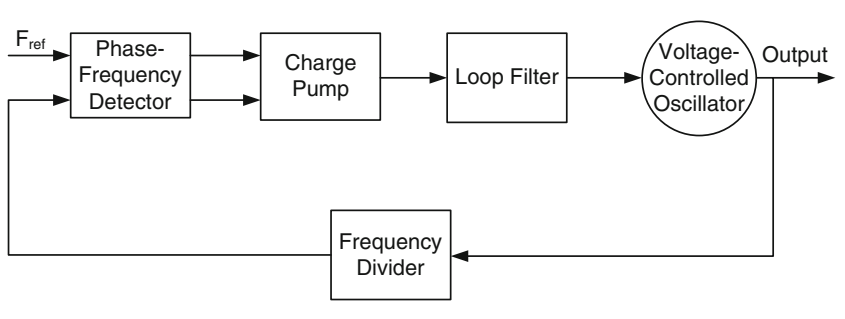

Fig. 14 General block diagram of charge pump PLL

a desired phase transfer function, as described in [1]. The IBM cmrf8sf $130 \mathrm{~nm}$ process was chosen as the technology for fabrication.

\subsubsection{Transistor-Level Design}

After the phase transfer function of the PLL has been determined, each component of the PLL was designed and implemented at the transistor level using Cadence. A general block diagram of the components comprising a typical charge pump PLL is shown in Fig. 14.

Phase-Frequency Detector The role of a phasefrequency detector (PFD) is to compute the phase and frequency error between the input reference signal and the voltage-controlled oscillator output. This information is delivered in terms of up/down pulses; this implies that there are three states, up, down, and no output. A fourth possible state, both outputs on, is an invalid state and triggers a reset through an AND gate. A more through description of how PFDs operate can be found in [13]. The PFD used is implemented with two D flip-flops, as shown in Fig. 15. The upper output is "up_bar" due to the specific implementation of the charge pump, described in more detail in the next section. The "down" output has a non-inverting buffer in order to somewhat equalize the delay introduced by the inverting buffer needed to invert the "up" signal.

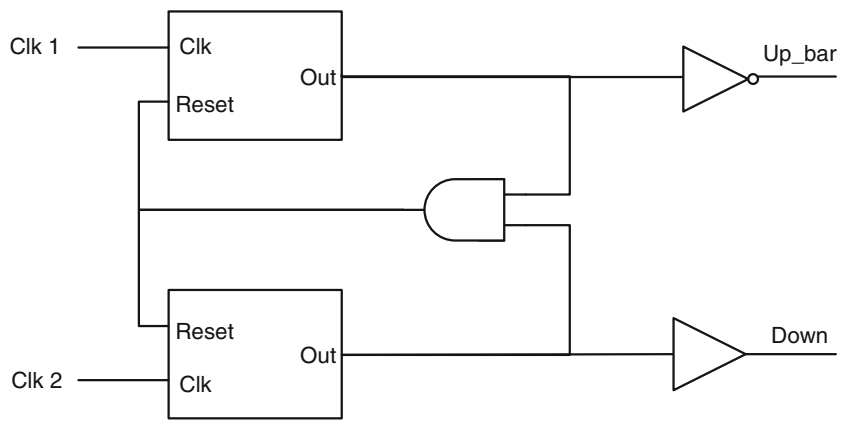

Fig. 15 Block diagram of PFD

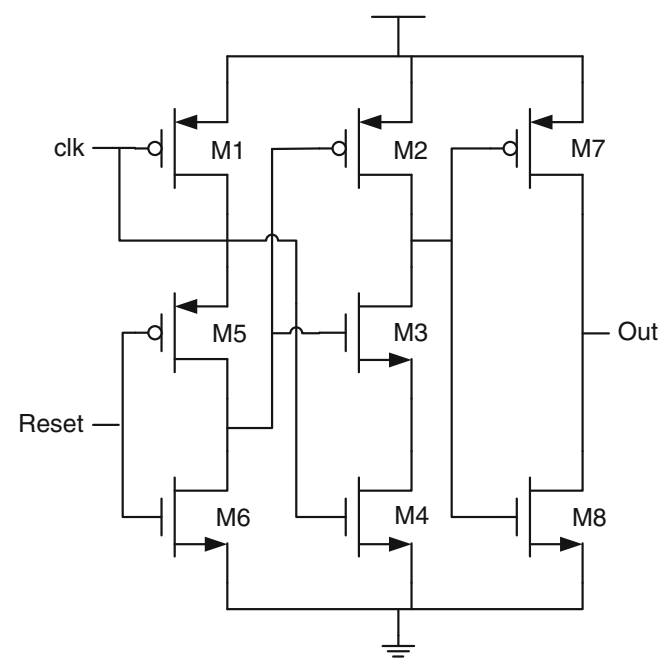

Fig. 16 Schematic of modified TSPC D flip-flop [9]

A true single-phase clocked (TSPC) based D flipflop [9] was used in this design because of the higher speeds achievable than pure CMOS logic. TSPC also has further benefits than typical precharged logic because, as its name implies, it requires only one clock phase as opposed to two. This contributes to simpler clock distribution and layout [12]. The positive-edge triggered D flip-flops [9] in Fig. 16 are modified to include a reset and feature a shorter path from input to output for shorter propagation delay and faster operation.

Charge Pump The charge pump translates pulses from the PFD into single-ended current for input into the loop filter. The charge pump design chosen is of the switch-at-source type, which allows the charge pump to switch faster as the switching transistors (connected to directly to the PFD output) are connected only to the source of one transistor, resulting in less parasitic capacitance [15]. This design, the current-matching charge pump [19], is shown in Fig. 17. It utilizes negative feedback to adjust the up/down branch reference currents according to the output voltage, resulting in less current mismatch. It also does not require an error opamp, reducing the required die space. Transistors M11-M16 form a current mirror to supply the reference current, with an input bias voltage (Vref_CP). For sizing, longer transistors are used to reduce channellength modulation effect. Common centroid layout was used wherever possible to aid in matching.

Voltage-Controlled Oscillator The voltage-controlled oscillator (VCO) translates voltage from the output of the loop filter into a corresponding frequency. As the 


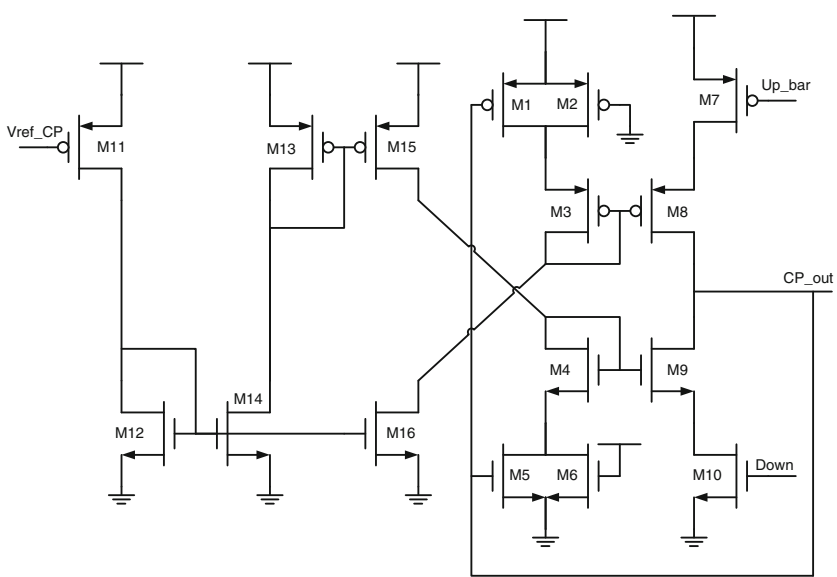

Fig. 17 Schematic of charge pump [10]

intent of the PLL is to demonstrate the concept of frequency generation via software-based sigma-delta modulation, the encoding may require as much as $25 \%$ tuning range. In order to meet this need, a ring oscillator topology was chosen for the VCO. As a high operational frequency is desired, a somewhat more complicated delay cell than an inverter would have to be used. Here, a multiple-pass ring oscillator-based VCO [3] was chosen. The block diagram showing how the delay cells are connected is shown in Fig. 18, while the schematic of an individual delay cell can be found in Fig. 19. The delay cell is differential in nature, with a single-ended control voltage. It also has two inputs that are connected to the previous stage $(\mathrm{p}+, \mathrm{p}-)$, and two inputs that are connected to the outputs that are two stages before the current one $(\mathrm{s}+, \mathrm{s}-)$. This allows it to work much like precharged logic; the output node is already partially charged when the input from the previous stage goes high. Three delay cells are used, which is the minimum required for oscillation to occur. This allows for maximum oscillation frequency. RF transistors from the cmrf8sf library were used. The VCO is extremely sensitive to layout (most likely due to parasitics); three iterations had to be completed before proper operation was achieved.

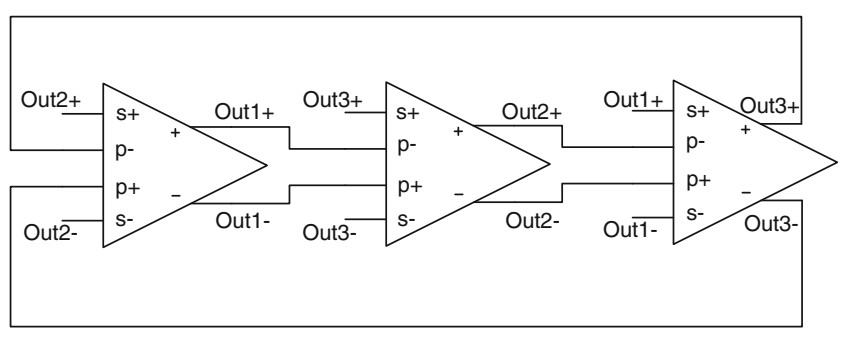

Fig. 18 Multiple-pass VCO block diagram [3]

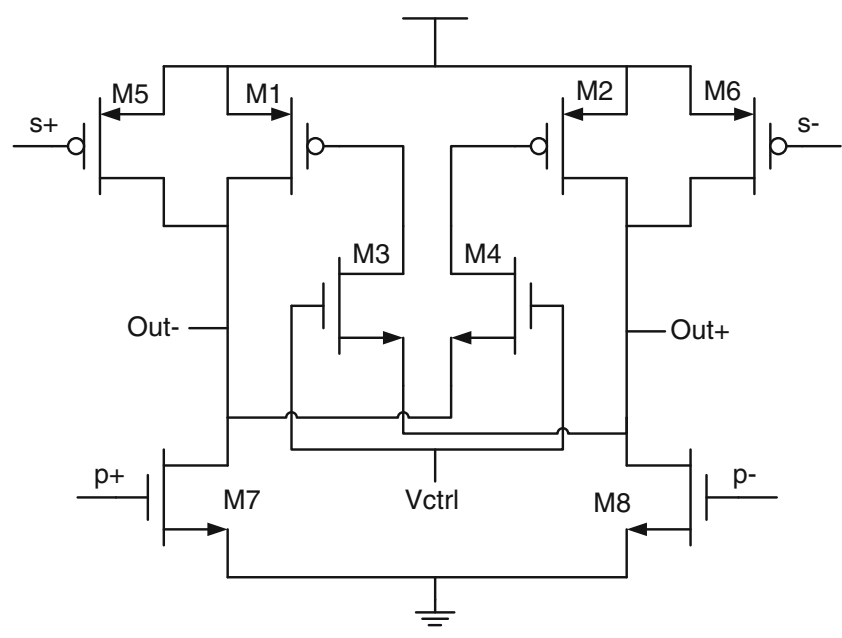

Fig. 19 Schematic of VCO delay cell [3]

Frequency Divider The basic frequency divider topology used is based on a D flip-flop with its inverting output fed back to the input. This has a frequency division ratio of two. The D flip-flip is implemented in a master-slave fashion with two latches. One latch takes as input an unmodified clock, and the other an inverted clock. This allows the resulting flip-flop to be edge-sensitive [12]. A block diagram of a differential implementation of the frequency divider can be found in Fig. 20.

Initially, all frequency dividers were planned to be TSPC-based. However, simulations showed that this design was not fast enough for the target speed of the voltage-controlled oscillator (roughly $10 \mathrm{GHz}$ ). Therefore, a modified current-mode logic (CML) latch-based divider [22] was used to halve the frequency before the TSPC-based frequency dividers. This modified CMLbased frequency divider, first introduced in [14], differs from traditional CML latches mainly in that it has an active load as opposed to a resistive load. It also removes the tail current sources to reduce transistor stacking,

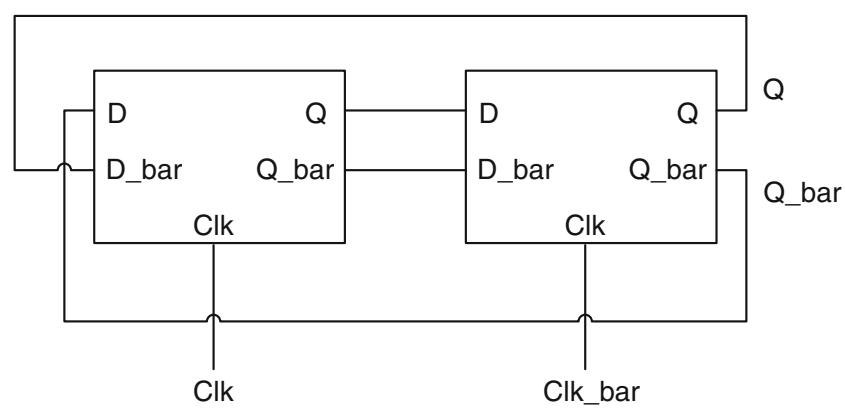

Fig. 20 Block diagram of frequency divider [14] 


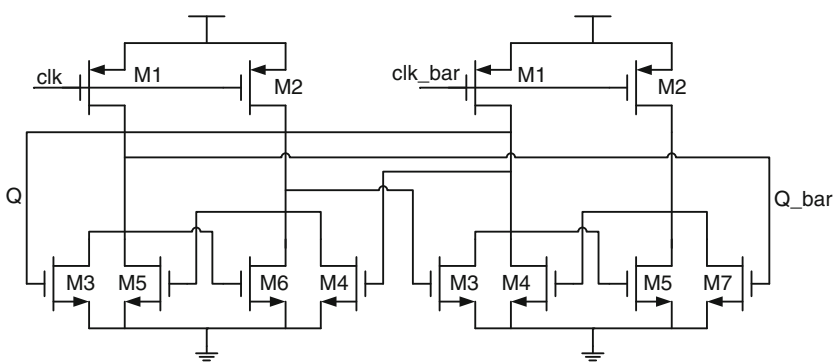

Fig. 21 Schematic of CML frequency divider [22]

making it more suitable for low supply voltages $(1.2 \mathrm{~V}$ in the case of IBM 130nm cmrf8sf) of current-day submicron technologies. To achieve the designed division ratio of 64 , one CML divider was used, followed by five TSPC dividers. The TSPC dividers were preferred because of their lower current draw and smaller die space requirement. The schematic of the CML divider and of the TSPC latch that the divider is based on can be found in Figs. 21 and 22, respectively.

Loop Filter The loop filter is essential for the PLL to accurately represent the phase transfer function as designed with the top-down method. The transfer function of the loop filter is third order and is as follows.

$$
F(s)=\frac{2.405 \times 10^{11} s+6.182 \times 10^{17}}{0.0153 \times s^{3}+1.771 \times 10^{5} s^{2}+1.62 \times 10^{12} s}
$$

Initially, the loop filter was planned to be implemented on chip. A passive, LC-ladder based approach was first considered; however, restrictions on zero placement made it unsuitable for the transfer function of the filter. Gm-C filters were evaluated next as an option. For a filter of with a bandwidth of around

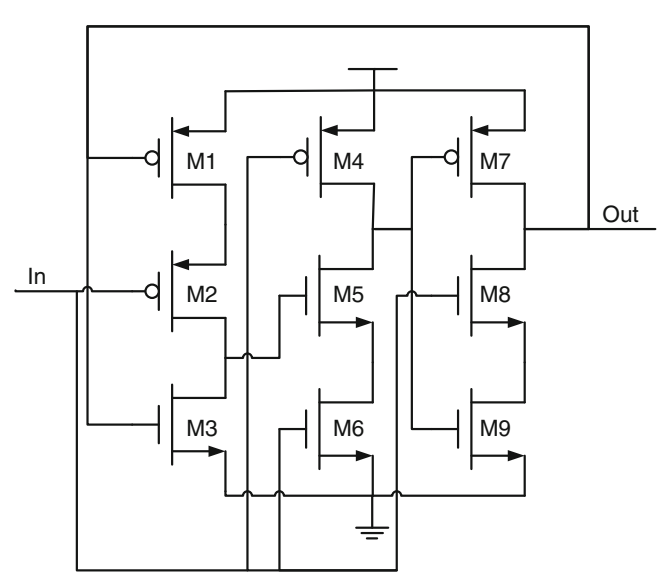

Fig. 22 Schematic of TSPC latch [12]

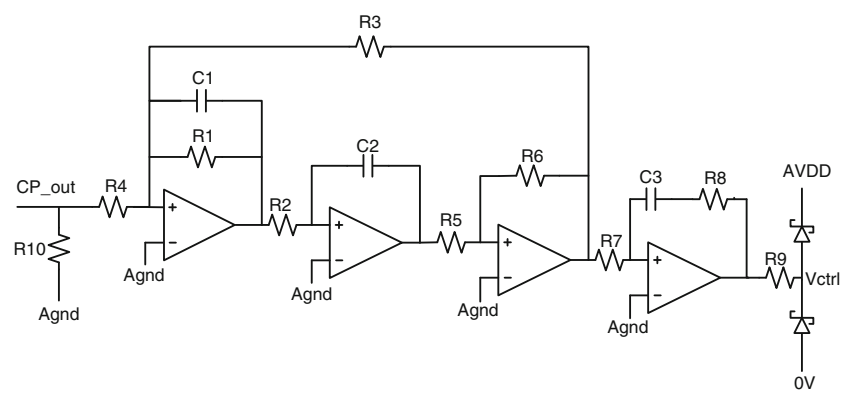

Fig. 23 Loop filter schematic $[4,17]$

$1 \mathrm{MHz}$, the gm values required from each cell would be on the order of $1 \times 10^{-6}$ to $1 \times 10^{-7}$. This is difficult to achieve in a gm-cell, as the bias currents would have to be correspondingly small as well. For similar reasons, an active RC implementation would be less than ideal, due to the large RC constants (and capacitors) that would be required. Switched-capacitor filters were considered, but with time restrictions imposed by the tape-out deadline, it was decided to move the loop filter off-chip.

The 3rd order off-chip filter (as shown in Fig. 23) is implemented using active RC, with a cascade of a TowThomas biquad and a first-order integrator section. The second section is required to implement a pole at DC with an additional zero (leaky integrator). It has a transfer function $\left(G(s)=G_{1}(s) G_{2}(s)\right)$ that consists of two parts,

$$
\begin{aligned}
G_{1}(s) & =\frac{1.332 \times 10^{14}}{s^{2}+1.133 \times 10^{7} s+1.037 \times 10^{14}} \\
G_{2}(s) & =\frac{3.891 \times 10^{-7} s+1}{3.367 \times 10^{-6} s}
\end{aligned}
$$

whose product is equal to Eq. 12. Comparing each of these transfer functions to the symbolic transfer functions of the two filter sections, i.e.

$$
\begin{aligned}
G_{1}(s) & =\frac{-\frac{1}{R_{2} R_{4} C_{1} C_{2}} s}{s^{2}+\frac{1}{R_{1} C_{1}} s+\frac{1}{R_{2} R_{3} C_{1} C_{2}}} \\
G_{2}(s) & =\frac{-R_{7} C_{3} s+1}{R_{8} C_{3} s}
\end{aligned}
$$

the resistor and capacitor values can be derived. The reference resistor value is set at $10 \mathrm{k} \Omega$ to reduce the current needed to drive the filter, as the charge pump has limited current drive capability. As such $R_{1}, R_{3}$, and $R_{4}$, were chosen to be this value, and $C_{1}$ was chosen 
to be $10 \mathrm{pF} . R_{2}$ and $C_{2}$ were then computed from the corresponding coefficients. Similarly, $C_{3}$ was chosen to be $120 \mathrm{pF}$ for the last stage, and $R_{7}$, and $R_{3}$ calculated. At the input of the filter, there is a resistor connected to analog ground $(0.6 \mathrm{~V})$ to allow the charge pump output to swing around the reference. Present at the output are a pair of Schottky diodes, which prevent damage to the VCO by limiting the range of the output voltage to $0-1.4 \mathrm{~V}$. Higher swing is possible because the opamps used for the filter (AD8045) are powered from $\pm 5 \mathrm{~V}$.

Input/Output Circuitry The PLL contains input/ output circuitry on-die to aid in interfacing to off-chip sources and equipment. The input has a level shifter [12] that accepts a $3.3 \mathrm{~V}$ signal and shifts it down to $1.2 \mathrm{~V}$ for the PFD input. The $3.3 \mathrm{~V} \mathrm{I} / \mathrm{O}$ (thick-oxide) transistors used at the input of the level shifter also has a larger maximum allowed $V_{\mathrm{DS}}$ before breakdown than standard $1.2 \mathrm{~V}$ transistors, allowing for a larger margin of error when connecting or setting up input sources. The level shifter also passes a $1.2 \mathrm{~V}$ input signal. The circuit diagram can be found in Fig. 24.

The output drivers consist of a chain of differential CML buffers. The transistor sizes of the chain is tapered (from small to large) so that a relatively large off-chip load could be driven with a reasonable propagation delay. This concept is similar to sizing a chain of inverters for minimum delay [12]. A total of fives stages were used. The driver was tested with a $50 \Omega$ terminated, $20 \mathrm{pF}$ load. This results in an approximate $200 \mathrm{mV}$ swing. Due to the large currents required to drive this load, the resistors used had to be carefully sized to ensure they would be able to handle the amount of current required. These polysilicon resistors were chosen because of their low variability over voltage and

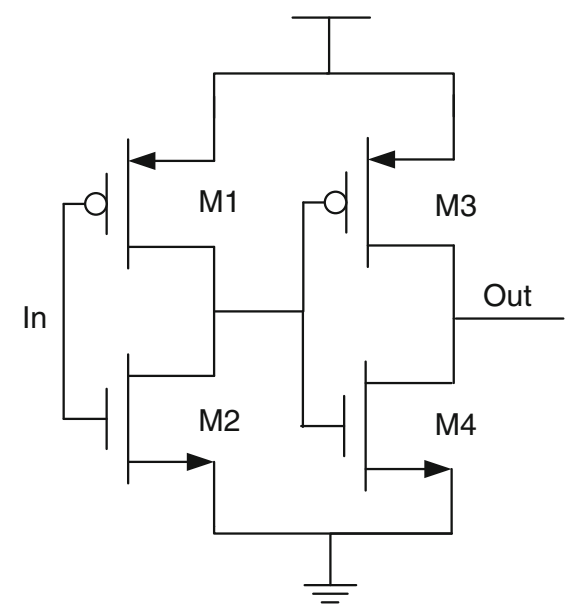

Fig. 24 Input buffer schematic [21]

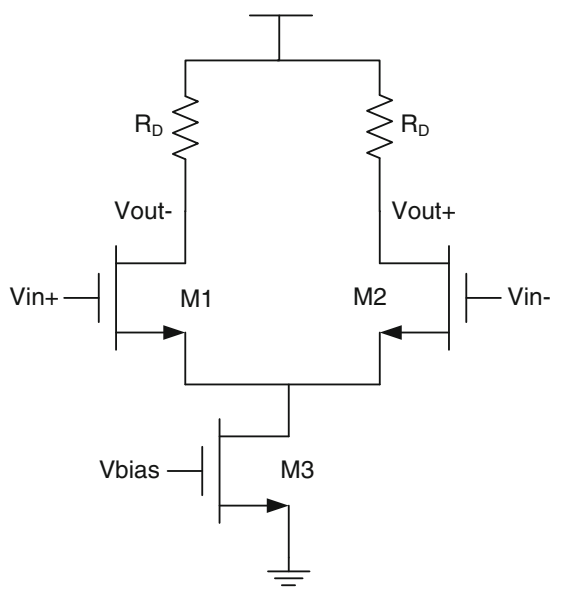

Fig. 25 CML buffer schematic [7]

temperature. The schematic of a single CML buffer stage can be found in Fig. 25.

\subsection{PCB Considerations}

In order to interface between the PLL to off-board equipment and instrumentation, a printed-circuit board (PCB) must be designed and fabricated.

Chip-Bonded PCB The PCB is intended to host the PLL die as well as the loop filter. It incorporates design features which aid in high-speed operation, such as microstrips, 50 impedance SMA connectors, and Rogers 4003 material (for greater dielectric uniformity).

Input/Output The input and output of the PCB are equipped with SMA connectors. The input is terminated with a $50 \Omega$ resistor. The high-speed differential outputs, however, needed a transmission line to be correctly terminated at high frequencies. Striplines and microstrips were considered; microstrips were chosen because striplines would be very difficult to debug due to their embedded nature. Rogers 4003 was selected as the dielectric material, which has $\varepsilon_{r}$ of 3.55. The dielectric thickness is 8 mils. Based on this information, the following equations from [11] were used to calculated the characteristic impedance $Z_{o}$, i.e.,

$Z_{o}=\frac{120 \pi}{\sqrt{\varepsilon_{e}}[W / d+1.393+0.667 \ln (W / d+1.444)]}$

where

$\varepsilon_{e}=\frac{\varepsilon_{r}+1}{2}+\frac{\varepsilon_{r}-1}{2} \frac{1}{\sqrt{1+12 d / w}}$

The width of the line was found to be 17 mils, which gives a $Z_{o}$ of approximately $51.9 \Omega$. These microstrips 


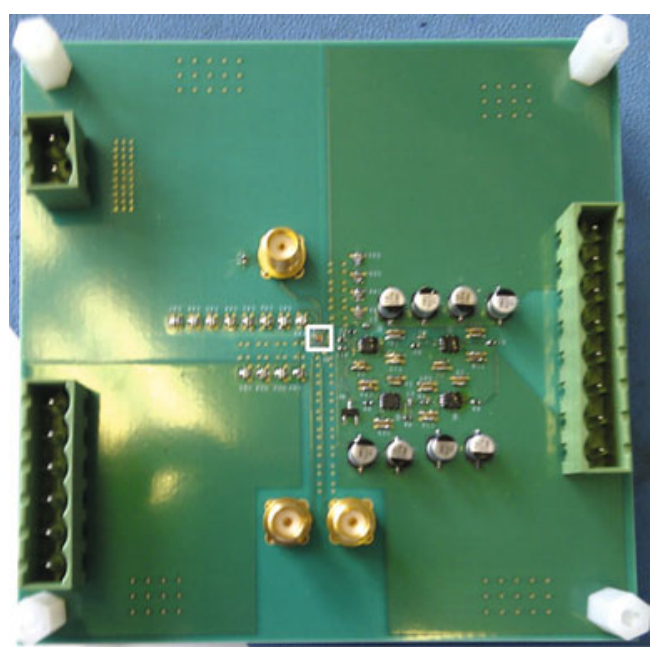

Fig. 26 Front of PCB with PLL die marked using white square at centre of diagram

were selected for impedance control for manufacturing, so a test coupon was created to measure their impedance. The test report states an impedance of $45.97 \Omega$ on average, which is close to the desired value.

Component Selection Due to concerns about the charge pump drive capability, efforts were made to minimize the parasitic capacitance offboard. To this end, package style of 0402 components were used, as well as the LFCSP package for the AD8045 opamp. Snap-on terminal blocks are used for the power connections to allow for quick connection and disconnection to the power supply, allowing for quick changes to be made to the PCB. Nylon standoffs were used on the corners of the PCB to reduce the chances of accidental shorts due to a messy workbench.

Die Bonding Normally, the die would be bonded to a package with pins using bondwires. Flip-chip ball grid array (BGA) type packaging would be ideal as it has no bondwires, but it is very expensive (above chip fabrication cost). Therefore, it was decided to bond the die to the PCB itself. This would eliminate the additional parasitics of a package (excluding bondwires). This process is often referred to as COB (chip-on-board). The final board with bonded die can be seen in Fig. 26 . The bonded die is marked with a white box in Fig. 26.

\section{Experimental Results}

Using the PCB with bonded PLL die, the functionality and performance of the phase and frequency signal

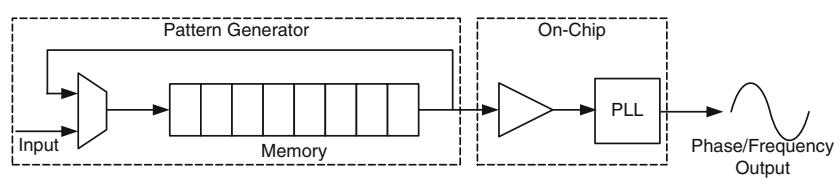

Fig. 27 Phase/frequency generation system test setup

generation system is explored. Some of these results were partially presented in [20]; the test results are presented in their entirety here.

\subsection{Test Setup}

In order to implement the phase/frequency generation system, a register or some other kind of memory is required. A sigma-delta bitstream is stored in this memory and looped to create a continuous bitstream. On-chip, an existing IEEE 1149.1 bus can be utilized to receive data from off-chip and store the data into a register that is routed to the components under test. A phase-locked loop is then used as a reconstruction filter as well as provides a means of scaling up the output frequency. Figure 27 shows the way the system is represented by the test setup. The sigma-delta modulator and the DFC/DTC are implemented in software, and the resultant output is looped to create an uninterrupted, continuous bitstream. As this is identical to the simulation setup, the bits are saved from Matlab and loaded into a Hewlett Packard 81130A pattern generator. It is programmed over GPIB via a Perl script, which configure the operating parameters (such as voltage levels, bit rate, etc.) as well as load the bits into memory. Time domain measurements were captured with

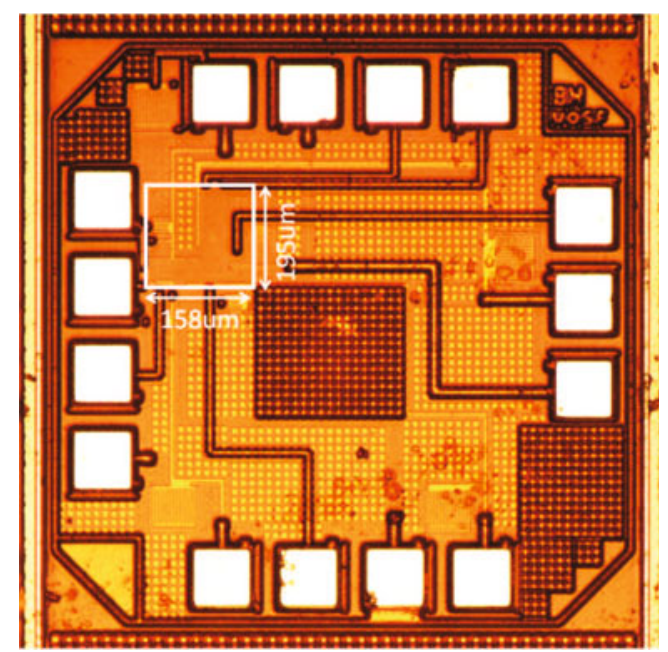

Fig. 28 PLL die micrograph 


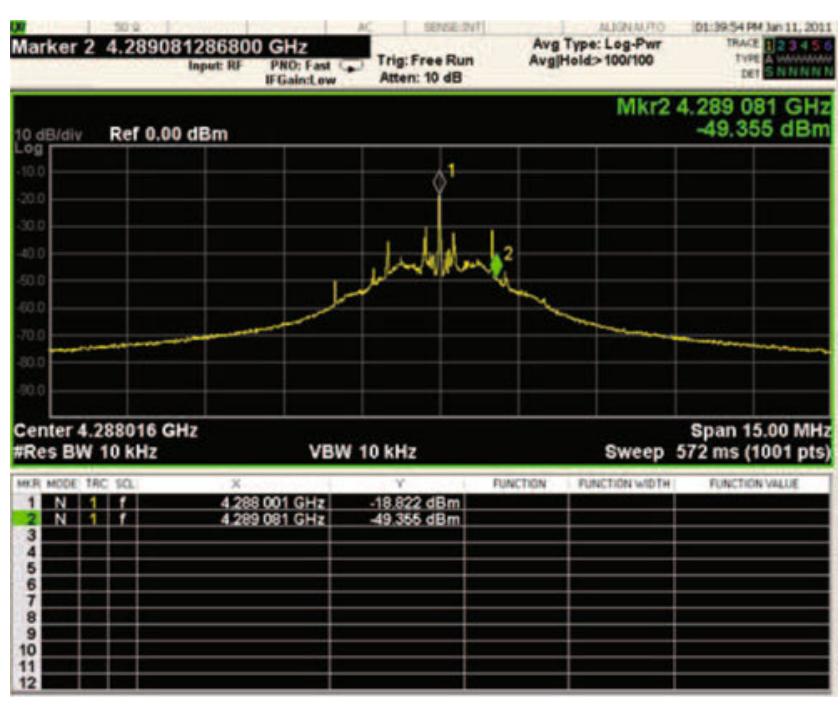

Fig. 29 PSD of PLL output with $67 \mathrm{MHz}$ clock input

an Agilent 1169A active differential probe connected to an Agilent Infiniium DSA80000B oscilloscope, while frequency domain measurements were obtained with the output connected directly to an Agilent MXA N9020A spectrum analyzer. The power supplies used were Agilent E3649A for all rails except analog ground, in which case the Hewlett Packard 6633A was used. The 6633A is able to sink current, which is important as the output signal of the charge pump is designed to swing around analog ground.

The PLL die micrograph is shown in Fig. 28. The die is $1 \mathrm{~mm} \times 1 \mathrm{~mm}$, and the core PLL area (including output driver) is about $158 \mu \mathrm{m}$ by $195 \mu \mathrm{m}$.

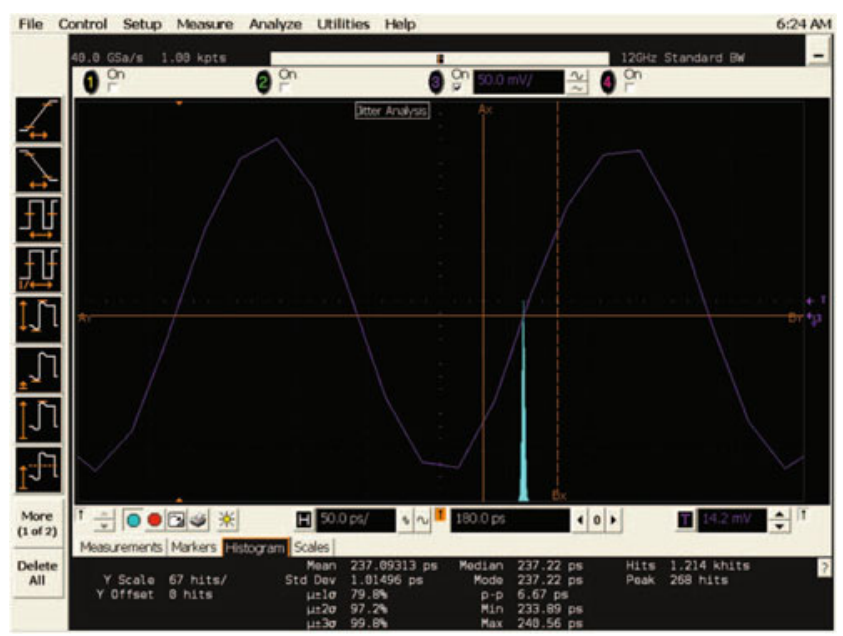

Fig. 30 Cycle-to-cycle jitter of PLL output for $65 \mathrm{MHz}$ clock input
Table 1 Clock measurement results

\begin{tabular}{ll}
\hline Parameter & Result \\
\hline Lock/Capture range @ 1.2 V & $59 \mathrm{MHz}-69 \mathrm{MHz}$ \\
Lock/Capture range @ 1.3 V & $72.5 \mathrm{MHz}-80 \mathrm{MHz}$ \\
Phase noise, 67 MHz input & $-70 \mathrm{dBc} / \mathrm{Hz} @ 1 \mathrm{MHz}$ \\
Cycle-to-cycle jitter, 67 MHz input & $1.02 \mathrm{ps}$ \\
\hline
\end{tabular}

\subsection{Clock Input}

Prior to testing frequency and phase signal generation, some basic PLL functionality has to be verified. For this purpose, the aforementioned pattern generator and an Agilent 33250A function generator was used to drive the PLL input with a clock. The function generator was used to sweep the input to check the lock and capture range of the PLL. It was found with the VCO powered from $1.2 \mathrm{~V}$, the capture range is around $59 \mathrm{MHz}$ to $69 \mathrm{MHz}$ (3.776-4.416 GHz output). With the VCO being powered from $1.3 \mathrm{~V}$, the capture range is $72.5 \mathrm{MHz}$ to $80 \mathrm{MHz}$ (4.640 GHz-5.120 GHz output). The lock range is found to be similar. This operational region is stable across several samples tested (three dies/boards). After this region, increasing the input frequency results in output from the VCO that is distorted and nonsinusoidal. However, it can observed that the PLL is tracking the input frequency on the spectrum analyzer as the output frequency is changing. Increasing the PLL input frequency past this region usually results in a narrow band of operation that locks with a sinusoidal output; however, the location of the region varies with the die tested (between 5 and $6 \mathrm{GHz}$ roughly). Further tests are performed in the 59-69 $\mathrm{MHz}$ input range, as this is the widest region of operation and is constant across different dies.

The phase noise is measured with a clock for different frequencies. The spectrum is captured using the spectrum analyzer, averaged, and the phase noise calculated using

$L\{\Delta \omega\}=10 \log _{10}\left(P_{\text {carrier }}\right)-10 \log _{10}\left(\frac{P_{\text {noise }} @ \Delta \omega}{\operatorname{Res} B W}\right)$.

Table 2 Frequencies generated

\begin{tabular}{lll}
\hline DC level & $\begin{array}{l}\text { DFC carrier } \\
\text { frequency } \\
(\mathrm{MHz})\end{array}$ & $\begin{array}{l}\text { PLL output } \\
\text { frequency } \\
(\mathrm{GHz})\end{array}$ \\
\hline 0.434 & 64.530 & 4.1299 \\
0.452 & 65.340 & 4.1818 \\
0.461 & 65.745 & 4.2077 \\
0.470 & 66.150 & 4.2336 \\
0.491 & 67.095 & 4.2941 \\
\hline
\end{tabular}




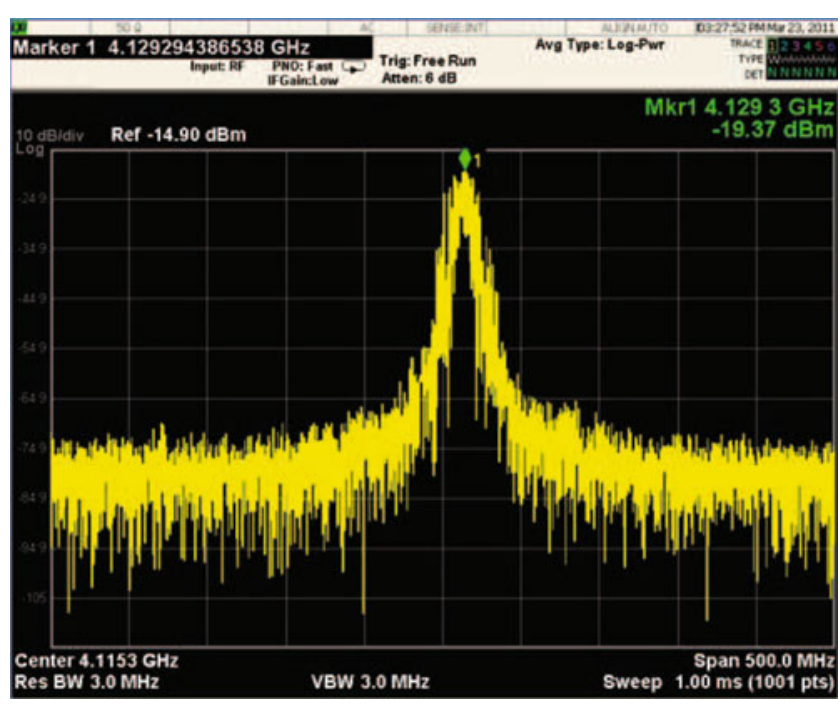

Fig. 31 Measured spectrum of PLL output for $0.434 \mathrm{~V}$ DC input

The results are between -70 and $-60 \mathrm{dBc} / \mathrm{Hz}$. A typical capture of the spectrum is shown in Fig. 29 for a 67 $\mathrm{MHz}$ input clock. The screen shows carrier power of $-18.822 \mathrm{dBm}$, noise power of $-49.355 \mathrm{dBm}$ at $1 \mathrm{MHz}$ offset, and resolution bandwidth of $10 \mathrm{kHz}$, giving an output phase noise of about $-70 \mathrm{dBc} / \mathrm{Hz} @ 1 \mathrm{MHz}$ offset. A time domain plot of the PLL output with a $65 \mathrm{MHz}$ clock input is shown in Fig. 30. It can be seen the output is sinusoidal-like. The plot also show the cycle-to-cycle jitter measured with a histogram one edge away from the trigger; the standard deviation as measured is $1.02 \mathrm{ps}$. The results from the clock input measurement tests are summarized in Table 1.

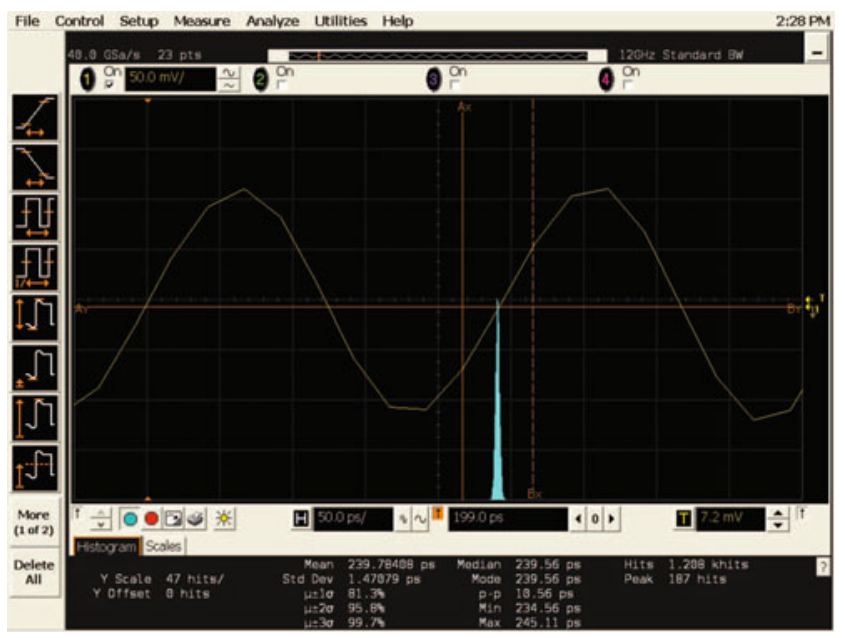

Fig. 32 Time domain capture of PLL output for 0.434V DC input

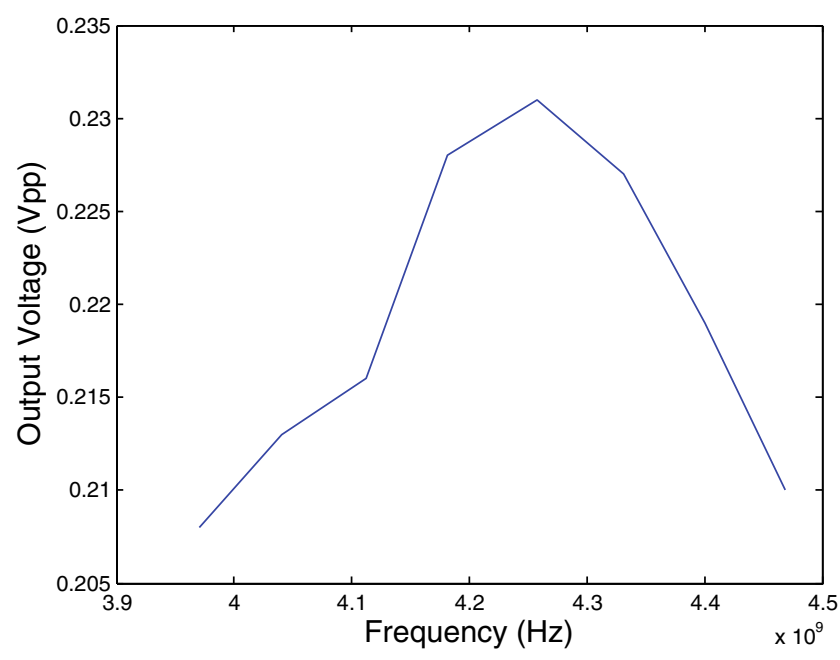

Fig. 33 Output voltage vs. freq. over $500 \mathrm{MHz}$ range centred at $4.2 \mathrm{GHz}$

\subsection{Frequency Signal Generation}

The PLL was used to synthesize signals of varying frequencies. The frequencies generated can be found in Table 2. Figure 31 shows the output spectrum of the PLL. The frequency is identical to that which we found in simulation (Fig. 12) and predicted by Eq. 9. The measured phase noise is $-42 \mathrm{dBc} / \mathrm{Hz}$ at $20 \mathrm{MHz}$ offset. Figure 32 shows a time domain capture of the sinusoidal PLL output. It also shows the cycle-to-cycle jitter as measured from the next rising edge from the triggered edge, which is approximately 1.48 ps. Figure 33 shows the output voltage (peak-to-peak) versus frequency over a $500 \mathrm{MHz}$ frequency range.

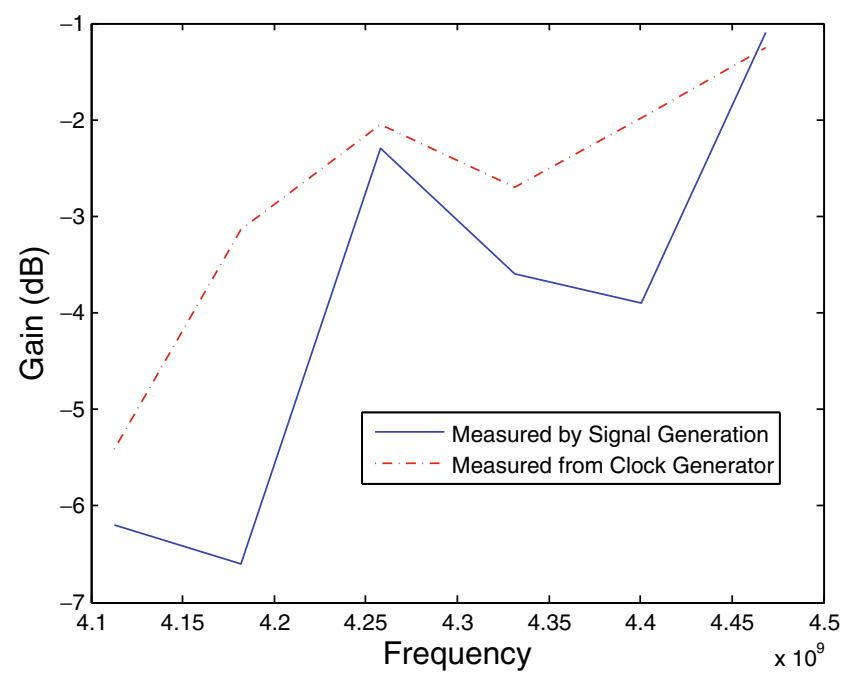

Fig. 34 Comparison of stripline characterization with frequency signal generator and external synthesizer 


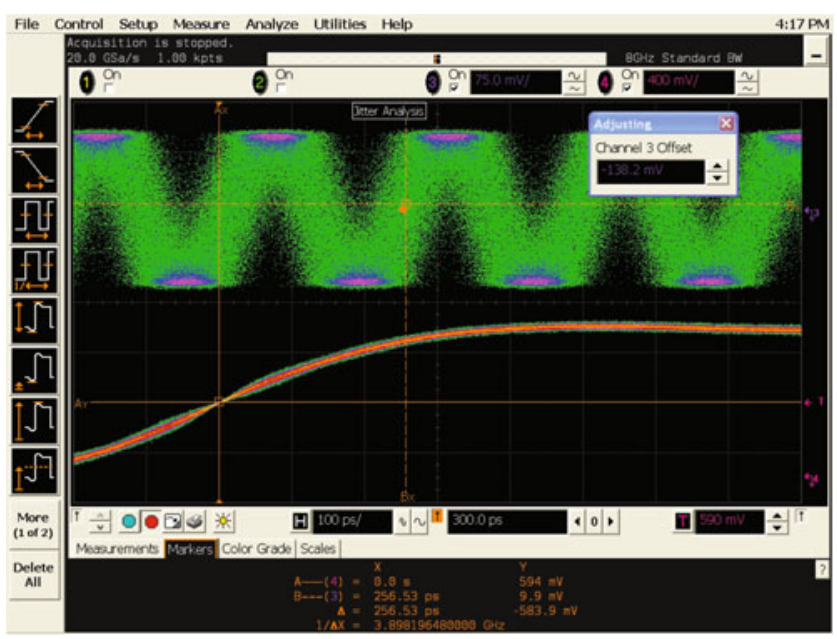

Fig. 35 Time domain PLL output with respect to reference clock, $0.434 \mathrm{~V}$ DC input

A potential application of frequency generation is testing for frequency response. Two off-chip microstrips, (the same as the ones used for differential output on the bonded die PLL on a solder sample PCB were connected in series. One end served as the input, the other the output. These microstrips were characterized with the frequency generator and the results compared with an off-chip source (a Centellax TG1C1-A clock synthesizer). The results correlate reasonably well. A comparison can be seen in Fig. 34.

\subsection{Phase Signal Generation}

The basic principle idea of phase signal generation is the ability to move an edge of a signal with respect to a reference signal. To demonstrate this, the pattern generator is loaded with various phase-encoded sigmadelta bitstreams with different DC codes as input to the PLL. Figure 35 shows one example of the time domain PLL output. The signal is displayed with colour grade, showing how much the signal is moving. The reference clock is taken from another output of the

Table 3 Phase output results

\begin{tabular}{lcc}
\hline DC code & $\begin{array}{l}\text { Measured } \\
\text { offset }(\mathrm{ps})\end{array}$ & $\begin{array}{c}\text { Adjusted } \\
\text { offset }(\mathrm{ps})\end{array}$ \\
\hline 0.4302 & 234.30 & 234.30 \\
0.434 & 236.53 & 236.53 \\
0.443 & 281.00 & 40.6 \\
0.452 & 69.74 & 69.74 \\
0.461 & 121.99 & 121.99 \\
0.470 & 155.34 & 155.34 \\
0.491 & 228.73 & 228.73 \\
\hline
\end{tabular}

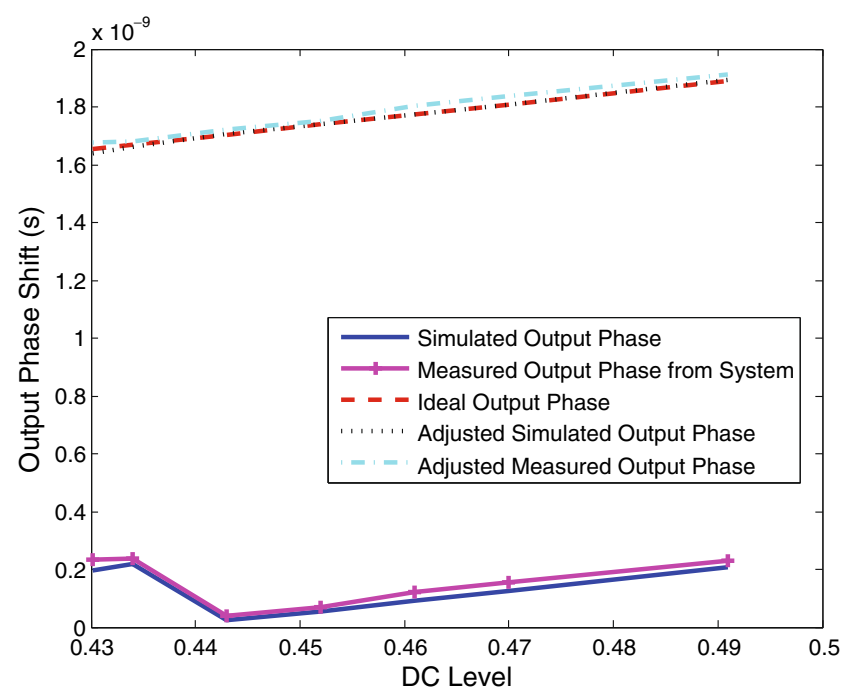

Fig. 36 Measured and simulated output phase vs. DC code

pattern generator. The delay in Fig. 35 is measured as 256.53 ps. Table 3 shows the delay for each input DC code. The adjusted offset is in case that the delay is greater than the period of the signal, in which case the period is subtracted from the measured delay value. When the delay grows greater than the period, the measured offset will decrease. This can be seen when the DC code is changed from 0.443 to 0.452 . As the output frequency is $4.16 \mathrm{GHz}$ (65 MHz input multiplied by 64), the period is 240.4 ps. The delay can be observed to be increasing with greater DC values. The measured and simulated delay versus the output DC code is shown in Fig. 36. The DC codes in Table 3

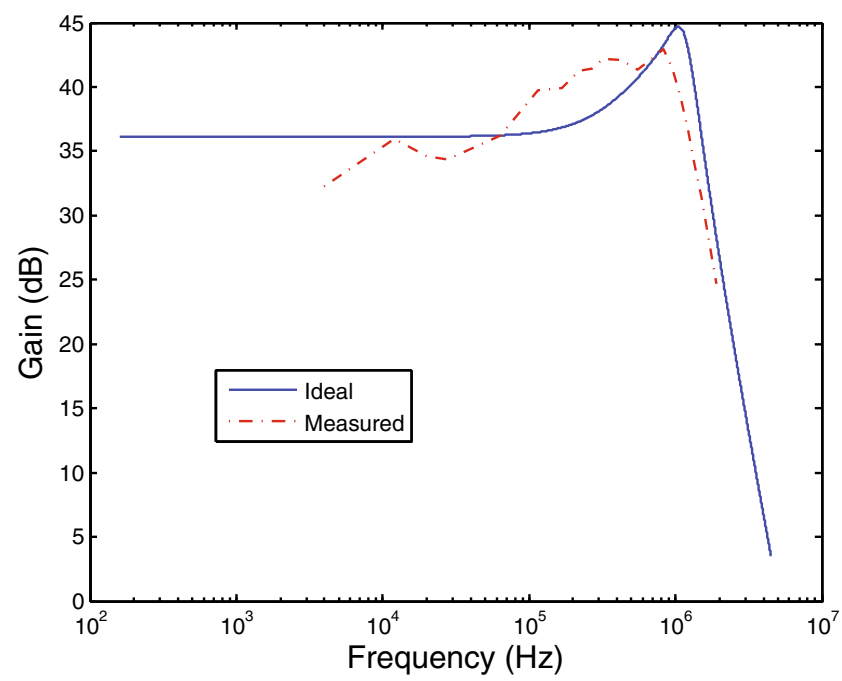

Fig. 37 Measured PLL jitter transfer function vs. ideal at $4.16 \mathrm{GHz}$ 
Fig. 38 Schematic of 2 nd order RC loop filter [16]

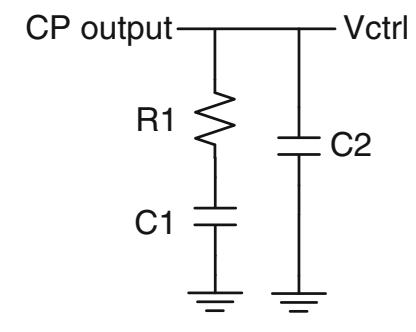

were used for both system measurements and Simulink. As can be observed from the line with ' + ' markers, the measured results are very close to Simulink results. Errors can be accounted for from the uncertainty in the measurements due to jitter, as well as additional delays introduced by cables and other measurement equipment. The adjusted measurement results can be seen with a dot-dash (.-) line, and closely tracks the simulated results.

To demonstrate a potential application of phase signal generation, the jitter transfer function of the PLL was characterized using sinusoidal phase-modulated signals [1]. The amplitude of the output phase with respect to the carrier was compared to the amplitude of the input signal applied to the sigma-delta modulator. A total of 22 points were taken. The carrier of the DTC output is at the $65 \mathrm{MHz}$ input, giving an output PLL carrier frequency of $4.16 \mathrm{GHz}$. The pattern generator sampling frequency is $260 \mathrm{MHz}$. The measured phase noise is $-55 \mathrm{dBc} / \mathrm{Hz}$ at $1 \mathrm{MHz}$ offset for a DC input code of 0.434 . The comparison between the ideal transfer function (solid line) versus the measured PLL transfer function (dashed line) can be found in Fig. 37. The correlation is reasonable.

\section{Future Research}

For future research, a 3rd order PLL with an on-chip, 2nd order integrated RC-filter would be investigated with regards to its performance and area. This would

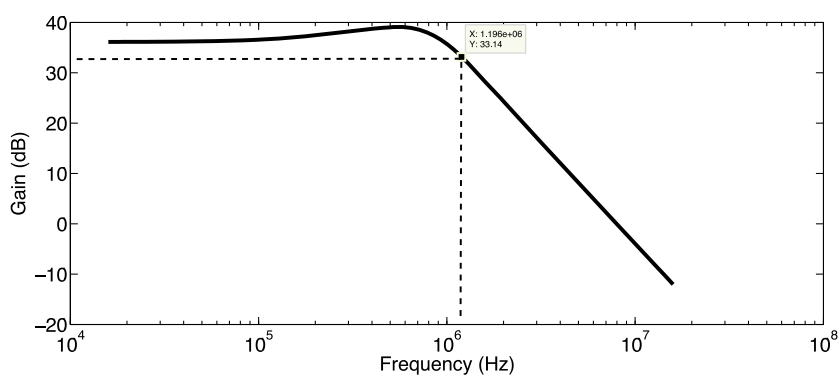

Fig. 39 Overall jitter transfer function of proposed 3rd order PLL aid in an analysis of the performance trade-offs between the advantages of having an integrated, lowerorder filter versus having a lower-order (and therefore lower SNR) sigma-delta modulator. For this implementation, the VCO gain is equal to $1 \mathrm{GHz} / \mathrm{V}$ and the charge pump current has a current of $125 \mu \mathrm{A}$. With respect to the loop filter, shown in Fig. $38, R_{1}$ is equal to $3 k \Omega C_{1}$ to $250 \mathrm{pF}$ and $C_{2}$ to $50 \mathrm{pF}$. Note that such component values are easily realizable on-chip. The overall jitter transfer function of the 3rd order PLL has a 1.2 MHz bandwidth and is shown in Fig. 39.

\section{Conclusion}

A phase/frequency signal generator for high-frequency applications amenable to digital testing methodologies without additional test pins was presented. The generator was implemented by means of a cyclic memory element and a custom integrated PLL. Although phase noise performance is much less than a fractional-N synthesizer, it is nevertheless useful for various debug and diagnosis situations that a design or test engineer may be in. In future research, a complete on-chip implementation with integrated loop filter will be investigated.

Open Access This article is distributed under the terms of the Creative Commons Attribution License which permits any use, distribution, and reproduction in any medium, provided the original author(s) and the source are credited.

\section{References}

1. Aouini S, Chuai K, Roberts GW (2011) A low-cost ATE phase signal generation technique for test applications. In: 2010 IEEE international test conference, Austin, TX, USA

2. Aouini S, Chuai K, Roberts GW (2012) Anti-imaging timemode filter design using a PLL structure with transfer function DFT. IEEE Trans Circuits Syst I 59:66-79

3. Eken YA, Uyemura JP (2004) A 5.9-GHz voltage-controlled ring oscillator in $0.18-\mu \mathrm{m}$ CMOS. IEEE J Solid-State Circuits 39:230-233

4. Gardner FM (2005) Phaselock techniques. Wiley, New York

5. Haurie X, Roberts GW (1996) A design, simulation and synthesis tool for delta-sigma-modulator-based signal sources. In: 1996 IEEE international symposium on circuits and systems, vol 4, Atlanta, GA, USA, pp 715-718

6. Hernandez L, Prefasi E (2008) Analog-to-digital conversion using noise shaping and time encoding. IEEE Trans Circuits Syst I Reg Pap 55:2026-2037

7. Heydari P, Mohanavelu R (2004) Design of ultrahigh-speed low-voltage CMOS CML buffers and latches. IEEE Trans Very Large Scale Integr (VLSI) Syst 12:1081-1093

8. IEEE (2001) IEEE standard for test access port and boundary-scan architecture. IEEE Std. 1149.1

9. Lee W-H, Cho J-D, Lee S-D (1999) A high speed and low power phase-frequency detector and charge-pump. In: Proc. of the Asia and South Pacific design automation conference, vol 1, Wanchai, Hong Kong, pp 269-272 
10. Lin T-H, Ti C-L, Liu Y-H (2009) Dynamic current-matching charge pump and gated-offset linearization technique for delta-sigma fractional-N PLLs. IEEE Trans Circuits Syst 56:877-885

11. Pozar DM (2005) Microwave engineering, 3rd edn. Wiley, New York

12. Rabaey JM, Chandrakasan A, Nikolic B (2003) Digital integrated circuits: a design perspective, 2nd edn. Pearson Education, Paris

13. Razavi B (2001) Design of analog CMOS integrated circuits. McGraw-Hill Education

14. Razavi B, Lee KF, Yan R-H (1994) A 13.4-GHz CMOS frequency divider. In: Digest of technical papers, 41st IEEE international solid-state circuits conference, San Francisco, CA, pp 176-177

15. Rhee W (1999) Design of high-performance CMOS charge pumps in phase-locked loops. In: Proc. of the 1999 IEEE international symposium on circuits and systems, vol 2, Orlando, FL, USA, pp 545-548

16. Rogers J, Plett C, Dai F (2006) Integrated circuit design for high-speed frequency synthesis. Artech House

17. Su K (2003) Analog filters, 2nd edn. Kluwer Academic, Dordrecht

18. Sunter S (2006) Advances in electronic testing: challenges and methodologies. In: Mixed signal testing and DFT. Springer, New York, pp 301-336

19. Ti C-L, Liu Y-H, Lin T-H (2008) A 2.4-GHz fractional-N PLL with a PFD/CP linearization and an improved CP circuit. In: Proc. of the 2008 IEEE international symposium on circuits and systems (Seattle, WA), p 1728

20. Tsai T-Y, Roberts GW (2011) Programmable phase/ frequency generator for system debug and diagnosis using the IEEE 1149.1 test bus. In: 2011 custom integrated circuits conference, San Jose, CA, USA

21. Wang W-T, Ker M-D, Chiang M-C, Chen C-H (2001) Level shifters for high-speed $1 \mathrm{~V}$ to $3.3 \mathrm{~V}$ interfaces in a $0.13 \mathrm{um} \mathrm{Cu}$ interconnection/low-k CMOS technology. In: Proc. of technical papers of international symposium on VLSI technology, systems, and applications, Hsinchu, Taiwan, pp 307-310

22. Wong K-LJ, Rylyakov A, Yang C-KK (2005) A broadband 44-GHz frequency divider in 90-nm CMOS. In: Compound semiconductor integrated circuit symposium, digest 2005, pp 196-199

23. Yang HY, Sarpeskar R (2005) A time-based energy-efficient analog-to-digital converter. IEEE J Solid-State Circuits 40:1590-1601
Tsung-Yen Tsai received the B.A.Sc. degree from the University of British Columbia, Canada, in 2008 and the M.Eng. from McGill University, Montreal Canada, in 2011. Since September 2011, he has been working as a junior design engineer at TSMC Design Technology Canada, Inc. in Ottawa, Canada.

Sadok Aouini received the B.Eng., M.Eng. and Ph.D. degrees from McGill University, Montreal Canada, in 2004, 2006 and 2011, respectively, all in electrical engineering. Since January 2011, he has been working as a mixed-signal design and test engineer at Ciena Co. in Ottawa, Canada. He is a member of the IEEE.

Gordon Walter Roberts received the B.A.Sc. degree from the University of Waterloo, Canada, in 1983 and the M.A.Sc. and $\mathrm{Ph}$.D. degrees from the University of Toronto, Canada, in 1986 and 1989, respectively, all in electrical engineering. At McGill University he is a full professor and holds the James McGill Chair in Electrical and Computer Engineering. He has co-written seven textbooks related to analog IC design and mixed-signal test. He has published numerous papers in scientific journals and conferences, and he has contributed chapters to various industrially focused textbooks. Dr. Roberts has held many administration roles within various conference organizations, such as the International Test Conference, Custom Integrated Circuit Conference, Design Automation Conference and the International Symposium on Circuits and Systems. He is the 2009 General Chair of the International Test Conference. Dr. Roberts is named on 8 patents, and has received numerous department, faculty and university awards for teaching test and electronics to undergraduates, and received several IEEE awards for his work on mixedsignal testing. Most notably, he has been awarded the 1992, 1993 and 2000 best or honorable mention papers at the International Test Conference. Several of his papers are published in the 2004 most significant papers published in the 35 years of ITC. Dr. Roberts has supervised over 40 graduate students at the masters and PhD level. In 2003 he took leave from McGill to cofound DFT Microsystems, Inc, a company specializing in highspeed timing measurement. He returned to McGill 2005 where his current research includes analog IC design methods and builtin selftest techniques for analog and high-speed digital circuits. Dr. Roberts is a Fellow of the IEEE. 